\title{
Padrões de cuidados em prevenção e tratamento de extrava- samento de antineoplásicos baseado em evidências clínicas
}

\author{
Patterns of care in prevention and treatment of clinical \\ evidence-based antineoplastic extravasation
}

\author{
Samya Raquel Soares Dias' • Fernanda Valéria Silva Dantas Avelino² $\bullet$ Elaine Cristina Carvalho Moura ${ }^{2}$ \\ Jéssica Pereira Costa ${ }^{2}$
}

\begin{abstract}
RESUMO
Objetivo: descrever padrões de cuidados em prevenção e tratamento de extravasamento de antineoplásicos baseado em evidências clínicas. Métodos: revisão integrativa da literatura realizada nas bases de dados Medline/PuBMed, $\mathrm{Cl}$ NAHL, LILACS e Science Direct. Resultados: foram localizados 30 estudos em inglês e espanhol, entre 2005 a 2015. Os temas predominantes quanto ao extravasamento são fatores de risco, medidas de prevenção e tratamento, como o uso de compressas e antídotos, estes foram organizados em quadros e classificados quanto ao nível de evidência e grau de recomendação. Conclusão: a prevenção é a principal estratégia. Ressalta-se a importância de implementação de protocolo assistencial.
\end{abstract}

Descritores: Antineoplásicos; Extravasamento de Materiais Terapêuticos e Diagnósticos; Enfermagem.

\begin{abstract}
Objective: to describe patterns of care in the prevention and treatment of clinical evidence based antineoplastic extravasation. Methods: integrative literature review carried out in Medline/PuBMed, CINAHL, LILACS and Science Direct databases. Results: 30 studies were conducted in english and spanish between 2005 and 2015 . The predominant themes regarding extravasation are risk factors, prevention and treatment measures, such as the use of compresses and antidotes, these were organized in tables and classified as level of evidence and degree of recommendation. Conclusion: prevention is the main strategy. It is important to emphasize the importance of implementing a care protocol.

Descriptors: Antineoplastic Agents; Extravasation of Diagnostic and Therapeutic Materials; Nursing.
\end{abstract}

'Enfermeira. Residente em Alta Complexidade, Universidade Federal do Piauí-UFPI,Teresina, Piauí, Brasil.Teresina-PI. Brasil. E-mail: samyaraquel02@hotmail.com. Autor correspondente.

${ }^{2}$ Enfermeira. Doutora. Docente da Universidade Federal do Piauí - UFPI, Teresina, Piauí, Brasil. 


\section{INTRODUÇÃO}

O câncer é considerado um problema de saúde pública mundial. Em 20I2, houve I4,I milhões de casos novos de câncer, 8,2 milhões de mortes por câncer e 32,6 milhões de pessoas vivendo com câncer (no prazo de 5 anos após o diagnóstico), a previsão é que esse seja um dado crescente. Destes, $57 \%$ dos casos novos, $65 \%$ das mortes e $48 \%$ dos casos de câncer de 5 anos prevalentes ocorreram nas regiões menos desenvolvidas ${ }^{(1)}$.

No que se refere ao tratamento, as três modalidades terapêuticas mais utilizadas são cirurgia, radioterapia e quimioterapia. Essas são usadas em conjunto, uma vez que atualmente, poucas neoplasias malignas são tratadas apenas com uma modalidade terapêutica. $A$ escolha depende do tipo de câncer, na reincidência, do estadiamento e da idade do paciente ${ }^{(2)}$.

O tratamento quimioterápico é age diretamente no processo de divisão celular por meio de substâncias químicas isoladas ou combinadas a partir de drogas chamadas antineoplásicas. Pode ser usado de forma paliativa, curativa, bem como nas formas adjuvante e neoadjuvante ${ }^{(3)}$.

Uma das preocupações decorrentes do tratamento quimioterápico é o extravasamento. Considerado um dano tecidual que decorre da fuga acidental do antineoplásicos para 0 tecido circundante ${ }^{(4)}$. $O$ extravasamento pode ser classificado em três grandes categorias com base no seu potencial de causar danos: os não-vesicantes, os irritantes e os vesicantes. $O$ primeiro pode causar uma ulceração, mas não evolui para necrose. Os irritantes podem causar dor no local e ao longo da veia, além da possibilidade de inflamação e ulceração em alguns casos. Já os vesicantes formam vesículas e ulcerações que quando não tratadas podem evoluir para necrose ${ }^{(5)}$.

Estima-se que o extravasamento é responsável por 0,5\% a 6,0\% dos eventos adversos associados com o tratamento. Quando considerado o quantitativo de eventos adversos associados ao tratamento de câncer, o número absoluto de extravasamento é bastante significativo(6-7).

Neste contexto, a atuação do enfermeiro frente aos antineoplásicos é assegurada no Brasil pelas Resoluções do Conselho Federal de Enfermagem 210 de 1998 e 257 de 201 I, que enfatizam que é função do enfermeiro elaborar protocolos terapêuticos de Enfermagem na prevenção, tratamento e minimização dos efeitos colaterais, além de preparar e administrar o quimioterápico antineoplásico ${ }^{(8-9)}$.

A assistência de Enfermagem requer referenciais teóricos que possam guiar o cuidado prestado. Dessa forma, a Prática Baseada em Evidências (PBE) permite ao enfermeiro aplicar a melhor evidência clínica frente à situação encontrada. Configura-se como uma forma coerente, segura e organizada de estabelecer práticas profissionais. Possibilita os melhores resultados e otimiza os recursos disponíveis, conforme a participação ativa de uma equipe multidisciplinar ${ }^{(10)}$.

Tendo em vista que a enfermagem apresenta um papel essencial na prestação de assistência oncológica, sendo responsável pela administração dos antineoplásicos e dos cuidados relacionados à mesma, este estudo possibilitou recomendações com o intuito de nortear a prática clínica a fim de reduzir ou minimizar riscos e danos que um extravasamento pode trazer aos pacientes, além de promover um cuidado uniforme por diferentes profissionais. Objetivou descrever padrões de cuidados em prevenção e tratamento de extravasamento de antineoplásicos baseado em evidências clínicas.

\section{MÉTODOS}

Trata-se de uma revisão integrativa da literatura sobre extravasamento de antineoplásicos. Para a construção foram seguidas as etapas propostas por Mendes; Silveira; Galvão( ${ }^{(I)}$ : identificação do tema, estabelecimento de critérios de inclusão e inclusão, categorização dos estudos, avaliação dos estudos, interpretação dos resultados e apresentação da revisão.

A busca foi realizada nas bases de dados: Medical Literature Analysis and Retrieval System Online (Medline/ PuBMed), Cummulative Index to Nursing and Allied Health Literature (CINAHL), Literatura Latino-Americana e do Caribe em Ciências da Saúde (LILACS) e Science Direct. Foi norteada pela seguinte questão de pesquisa: Quais as evidências e recomendações científicas diante do extravasamento de antineoplásicos?

$\mathrm{Na}$ identificação foram utilizados termos chaves/descritores e métodos de busca específicos para cada base, conforme quadro I.

Foram incluídos na pesquisa os estudos: aplicados em seres humanos; que abordem extravasamento de antineoplásicos vesicantes, irritantes e não irritantes; relacionados à prevenção e tratamento de extravasamento de quimioterápicos; publicados nacionais e internacionais; publicados em inglês, português ou espanhol; entre os anos de 2005 e 20I5; e estudos que atendam a clientela acima de 18 anos.

\section{RESULTADOS}

Com os termos chave foram encontrados 639 artigos. Após aplicação dos critérios de inclusão e exclusão foram selecionados 30 artigos, caracterizados quanto ao periódico, ano de publicação, tipo de estudo e objetivos (conforme tabela I). Destes II (36,67\%) artigos são do tipo estudo de caso, 8 (26,67\%) são revisões da literatura e $18(60 \%)$ foram publicados nos últimos cinco anos. 
QUADRO 1 - Bases, descritores e esquemas de buscas. Teresina-PI, 2016.

\begin{tabular}{|c|c|c|c|c|}
\hline Base & Medline & Cinahl & Science direct & Lilacs \\
\hline & MeshTerms* & Títulos Cinahl & $\begin{array}{c}\text { Descritores não } \\
\text { controlados }\end{array}$ & $\operatorname{Decs}^{* *}$ \\
\hline $\begin{array}{l}\text { Descritores/ } \\
\text { termos chave }\end{array}$ & $\begin{array}{c}1 \text { Antineoplastic } \\
2 \text { Agents } \\
3 \text { Extravasation of Diagnostic } \\
\text { and Therapeutic Materials } \\
4 \text { Therapeutics } \\
5 \text { Nursing Care }\end{array}$ & $\begin{array}{c}1 \text { Antineoplastic } \\
2 \text { Agents } \\
3 \text { Extravasation of } \\
\text { Diagnostic and Therapeutic } \\
\text { Materials }\end{array}$ & $\begin{array}{l}1 \text { Antineoplastic } \\
2 \text { Extravasation }\end{array}$ & $\begin{array}{l}1 \text { Antineoplásicos } \\
2 \text { Extravasamento }\end{array}$ \\
\hline \multirow[t]{2}{*}{$\begin{array}{c}\text { Esquemas de } \\
\text { busca }\end{array}$} & $\begin{array}{c}\text { (("Antineoplastic } \\
\text { Agents"[Mesh]) AND } \\
\text { "Extravasation of } \\
\text { Diagnostic and Therapeutic } \\
\text { Materials"[Mesh]) AND } \\
\text { "Nursing Care"[Mesh] }\end{array}$ & \multirow[t]{2}{*}{$\begin{array}{l}\text { Antineoplastic Agents AND } \\
\text { Extravasation of Diagnostic } \\
\text { and Therapeutic Materials }\end{array}$} & \multirow[t]{2}{*}{$\begin{array}{c}\text { Antineoplastic AND } \\
\text { Extravasation }\end{array}$} & $\begin{array}{c}\text { Antineoplásicos } \\
\text { AND Extravasamento }\end{array}$ \\
\hline & $\begin{array}{l}\text { Antineoplastic Agents AND } \\
\text { Extravasation of Diagnostic } \\
\text { and Therapeutic Materials }\end{array}$ & & & Antineoplásicos \\
\hline
\end{tabular}

Legenda: * Medical Subject Headings. ** Descritores em Ciências da Saúde.

\section{TABELA 1 - Caracterização dos artigos encontrados. Teresina-PI, 2016}

\begin{tabular}{|c|c|c|c|c|}
\hline Artigo & Periódico & Ano & Tipo de estudo & Objetivos \\
\hline A1 & $\begin{array}{l}\text { Seminars in Oncology } \\
\text { Nursing }\end{array}$ & 2011 & $\begin{array}{l}\text { Opinião de } \\
\text { especialista }\end{array}$ & $\begin{array}{l}\text { Apresentar uma atualização clínica sobre prevenção, detecção e } \\
\text { tratamento de extravasamento de quimioterapia vesicante baseada } \\
\text { em evidências. }\end{array}$ \\
\hline $\mathrm{A} 2$ & The Journal of Medicine & 2011 & Estudo de caso & Relatar tratamento de extravasamento de vinorelbine. \\
\hline A3 & $\begin{array}{l}\text { Japanese Journal of } \\
\text { Clinical Oncology }\end{array}$ & 2014 & Estudo quantitativo & Descrever características clínica de extravasamento. \\
\hline A4 & BMJ Case Reports & 2013 & Estudo de caso & Descrever tratamento de extravasamento de antraciclinas. \\
\hline A5 & BMJ Case Reports & 2013 & Estudo de caso & Descrever condutas em extravasamento de antraciclinas. \\
\hline A6 & The Journal Medicine & 2012 & Estudo de caso & Relatar tratamento de extravasamento após ciclo de quimioterapia. \\
\hline A7 & $\begin{array}{l}\text { European Journal of } \\
\text { Oncology Nursing }\end{array}$ & 2015 & Grupo controle & $\begin{array}{l}\begin{array}{l}\text { Validar instrumento de avaliação de dificuldade de inserção } \\
\text { intravenosa. }\end{array}\end{array}$ \\
\hline A8 & Surgical Oncology & 2012 & Estudo quantitativo & $\begin{array}{l}\text { Avaliar o índice de complicações em pacientes com cateter } \\
\text { totalmente implantado. }\end{array}$ \\
\hline A9 & $\begin{array}{l}\text { EJSO The Journal of } \\
\text { Cancer Surgery }\end{array}$ & 2015 & Série de casos & $\begin{array}{l}\text { Propor um procedimento estruturado para gestão do } \\
\text { extravasamento. }\end{array}$ \\
\hline A10 & $\begin{array}{l}\text { Annals Dermatology } \\
\text { Venereol }\end{array}$ & 2007 & Estudo de caso & $\begin{array}{l}\text { Descrever tratamento de extravasamento de cisplatina em cateter } \\
\text { totalmente implantado. }\end{array}$ \\
\hline A11 & $\begin{array}{l}\text { Journal of Plastic, } \\
\text { Reconstructive \& } \\
\text { Aesthetic Surgery }\end{array}$ & 2011 & Estudo de caso & Descrever a técnica de SWOP* para extravasamento. \\
\hline A12 & $\begin{array}{l}\text { Chinese Journal of } \\
\text { Traumatology }\end{array}$ & 2009 & Estudo de caso & $\begin{array}{l}\text { Apresentar a utilização da terapia à vácuo no extravasamento de } \\
\qquad \text { vinorelbine. }\end{array}$ \\
\hline A13 & Cancer Nursing Practice & 2011 & Estudo descritivo & $\begin{array}{c}\text { Descrever a técnica de lavagem com solução salina em } \\
\text { extravasamento. }\end{array}$ \\
\hline A14 & $\begin{array}{l}\text { Journal of The National } \\
\text { Cancer Institute }\end{array}$ & 2007 & Estudo de caso & Apresenta tratamento para extravasamento de antineoplásico. \\
\hline A15 & $\begin{array}{l}\text { Journal of Clinical } \\
\text { Oncology }\end{array}$ & 2009 & Estudo de caso & Relatar tratamento de extravasamento de Trabectedin. \\
\hline
\end{tabular}




\begin{tabular}{|c|c|c|c|c|}
\hline A16 & Cancer Nursing & 2005 & Estudo quantitativo & $\begin{array}{l}\text { Verificar a incidência de extravasamento de citotóxicos em } \\
\text { pacientes de um hospital de São Paulo. }\end{array}$ \\
\hline A17 & $\begin{array}{l}\text { European Journal of } \\
\text { Cancer Care }\end{array}$ & 2013 & Série de casos & $\begin{array}{l}\text { Apresentar série de casos de extravasamento de citotóxicos } \\
\text { tratados com solução salina. }\end{array}$ \\
\hline A18 & Annals of Oncology & 2012 & $\begin{array}{l}\text { Opinião de } \\
\text { especialistas }\end{array}$ & $\begin{array}{l}\text { Apresentar gestão de extravasamento de quimioterapia baseada } \\
\text { nas diretrizes clínicas da European Oncology Nursing Society. }\end{array}$ \\
\hline A19 & Farmacia Hospitalaria & 2012 & Revisão integrativa & $\begin{array}{l}\text { Apresentar atualizações de tratamentos de extravasamento por } \\
\text { agentes citostáticos. }\end{array}$ \\
\hline A20 & $\begin{array}{l}\text { European Journal of } \\
\text { Oncology Nursing }\end{array}$ & 2008 & $\begin{array}{l}\text { Opinião de } \\
\text { especialista }\end{array}$ & $\begin{array}{l}\text { Apresentar orientações sobre extravasamento baseados no } \\
\text { guideline da European Oncology Nursing Society. }\end{array}$ \\
\hline A21 & $\begin{array}{l}\text { Seminars in Oncology } \\
\text { Nursing }\end{array}$ & 2007 & $\begin{array}{l}\text { Opinião de } \\
\text { especialista }\end{array}$ & $\begin{array}{c}\text { Descrever mecanismos de lesão associada a vesicantes com } \\
\text { ligação de DNA e não vinculados ao DNA. Avaliar procedimentos } \\
\text { utilizados na prática clínica para gerenciar extravasamentos de } \\
\text { vesicantes. }\end{array}$ \\
\hline A22 & $\begin{array}{l}\text { Online Brazilian Journal } \\
\text { of Nursing }\end{array}$ & 2008 & Revisão integrativa & $\begin{array}{l}\text { Identificar na literatura evidências relacionadas com a prevenção } \\
\text { de extravasamento de quimioterapia vesicantes em pacientes com } \\
\text { infusão intravenosa periférica. }\end{array}$ \\
\hline A23 & $\begin{array}{l}\text { Clinical Journal of } \\
\text { Oncology Nursing }\end{array}$ & 2013 & $\begin{array}{l}\text { Opinião de } \\
\text { especialista }\end{array}$ & $\begin{array}{c}\text { Apresentar aos enfermeiros informações baseadas em evidências } \\
\text { úteis para eliminar ou reduzir a gravidade de uma lesão por } \\
\text { extravasamento quimioterapia. }\end{array}$ \\
\hline A24 & The Oncologist & 2008 & $\begin{array}{l}\text { Revisão de } \\
\text { literatura }\end{array}$ & $\begin{array}{c}\text { Apresentar aprovação de Dexrazoxane (Totect) para tratamento de } \\
\text { extravasamento de quimioterapia por antraciclina. }\end{array}$ \\
\hline A25 & $\begin{array}{l}\text { The International Journal } \\
\text { of Clinical Practice }\end{array}$ & 2013 & $\begin{array}{l}\text { Revisão de } \\
\text { literatura }\end{array}$ & $\begin{array}{c}\text { Demonstrar eficácia de Dexrazoxane (Savene) para } \\
\text { extravasamento de antraciclinas. }\end{array}$ \\
\hline A26 & Emergency Medicine & 2012 & $\begin{array}{l}\text { Revisão de } \\
\text { literatura }\end{array}$ & $\begin{array}{l}\text { Revisar na literatura o efeito de dexrazoxano na diminuição do } \\
\text { risco de necrose após extravasamento de antraciclina. }\end{array}$ \\
\hline A27 & $\begin{array}{l}\text { European Journal of } \\
\text { Cancer Care }\end{array}$ & 2015 & Revisão sistemática & $\begin{array}{l}\text { Analisar a qualidade das evidências que sustentam a prática } \\
\text { contemporânea e para determinar se a experiência do paciente é } \\
\text { levada em conta. }\end{array}$ \\
\hline A27 & $\begin{array}{l}\text { Clinical Journal of } \\
\text { Oncology Nursing }\end{array}$ & 2009 & $\begin{array}{l}\text { Opinião de } \\
\text { especialista }\end{array}$ & $\begin{array}{l}\text { Descrever as recomendações dos fabricantes, enumerar antídotos } \\
\text { e tratamentos aprovados pela FDA, e recomendações publicadas. }\end{array}$ \\
\hline A28 & Cancer Nursing Practice & 2009 & $\begin{array}{l}\text { Opinião de } \\
\text { especialista }\end{array}$ & $\begin{array}{l}\text { Descrever tratamentos baseados em evidências para necrose } \\
\text { tecidual causada por vesicantes. }\end{array}$ \\
\hline A29 & Oncology Nursing Forum & 2006 & $\begin{array}{l}\text { Revisão de } \\
\text { literatura }\end{array}$ & $\begin{array}{l}\text { Revisar a literatura sobre a incidência, prática, recomendações, } \\
\text { gerenciamento de enfermagem e lacunas no conhecimento } \\
\text { relevantes sobre extravasamento de vesicantes. }\end{array}$ \\
\hline A30 & Oncology Nursing Forum & 2006 & $\begin{array}{l}\text { Revisão de } \\
\text { literatura }\end{array}$ & $\begin{array}{c}\text { Sintetizar recomendações atuais, e discutir contradições a respeito } \\
\text { do tratamento de extravasamento de vesicantes. }\end{array}$ \\
\hline
\end{tabular}

Legenda: *Subcutaneous wash-out procedure.

A análise dos artigos foi feita por meio de um instrumento adaptado que possibilitou a formulação das evidências. Para tanto, as mesmas foram classificadas quanto ao nível de evidência conforme o referencial de Melnyk; Fineout-Overholt ${ }^{(12)}$ e grau de recomendação segundo referencial da National Pressure Ulcer Advisory Panel ${ }^{(13)}$. As evidências foram descritas nos quadros 2 e 3, por temas mais prevalentes, o primeiro descreve as medidas de prevenção e o segundo de tratamento.
Como existem técnicas terapêuticas diversas para tratamento de extravasamento, foi construído o quadro 4, que descreve os antídotos com dosagens e aplicação dos demais de cada tipo.

\section{DISCUSSÃo \\ Evidência Clínicas \\ Epidemiologia}

A ocorrência de extravasamento é rara, contudo acarreta danos. Estudo realizado em um Ambulatório de Qui- 
QUADRO 2 - Evidências sobre prevenção de extravasamento de antineoplásicos. Teresina-PI, 2016

\begin{tabular}{|c|c|c|c|}
\hline Temas & Recomendações & Evidência & $\mathrm{GR}^{*}$ \\
\hline \multirow{6}{*}{$\begin{array}{l}\text { Rede venosa } \\
\text { periférica }\end{array}$} & Avaliar a rede venosa periférica quanto à disponibilidade de veias. & IV & B \\
\hline & Evitar veias de pequeno calibre. & IV & $\mathrm{B}$ \\
\hline & Não infundir em veias tortuosas e esclerosadas. & IV & $\mathrm{B}$ \\
\hline & Conferir retorno venoso antes de retomar uma infusão pausada. & IV & $\mathrm{B}$ \\
\hline & Puncionar em veias de grande calibre no antebraço. & VI & C \\
\hline & Não palpar a veia no momento da punção. & VII & $\mathrm{C}$ \\
\hline \multirow{5}{*}{$\begin{array}{l}\text { Dispositivos para } \\
\text { punção }\end{array}$} & Não utilizar cateter agulhado. & IV & B \\
\hline & Fazer uso de cateter totalmente implantado. & $\mathrm{VI}$ & $\mathrm{C}$ \\
\hline & $\begin{array}{l}\text { Infusões e vesicantes com duração entre } 12 \text { e } 24 \mathrm{~h} \text { indicado cateter venoso } \\
\text { central. }\end{array}$ & VII & C \\
\hline & Utilizar cateteres de pequeno calibre nas infusões. & $\mathrm{VI}$ & C \\
\hline & Administrar vesicantes em bomba de infusão gota a gota. & $\mathrm{VI}$ & C \\
\hline \multirow{4}{*}{$\begin{array}{l}\text { Local da punção } \\
\text { periférica }\end{array}$} & $\begin{array}{l}\text { Dar preferencia ao antebraço, dorso da mão, punho e fossa antecubital, } \\
\text { respectivamente. }\end{array}$ & $\mathrm{VI}$ & C \\
\hline & Não administrar em extremidades inferiores. & VII & C \\
\hline & Não administrar na presença de edema. & VII & $\mathrm{C}$ \\
\hline & Conferir a localização do cateter venoso central & VII & $\mathrm{C}$ \\
\hline \multirow{7}{*}{$\begin{array}{l}\text { Assistência de } \\
\text { Enfermagem }\end{array}$} & Enfermeiro acompanhar a infusão a cada 30 min. & $\mathrm{VI}$ & $\mathrm{C}$ \\
\hline & Cessar a infusão caso o paciente precise se movimentar. & $\mathrm{VI}$ & $\mathrm{C}$ \\
\hline & Registrar ações em ficha de acompanhamento. & $\mathrm{VI}$ & C \\
\hline & $\begin{array}{l}\text { Lavar o acesso com } 10 \text { a } 20 \mathrm{ml} \text { de SF 0,9\% entre infusões de drogas } \\
\text { diferentes. }\end{array}$ & VII & $\mathrm{C}$ \\
\hline & $\begin{array}{l}\text { Infusões de vesicantes em bolus deve ser administrada concomitante à fluido } \\
\text { compatível. }\end{array}$ & VII & C \\
\hline & Avaliar aparecimento de sintomas: edema, dor, rubor e infusão lenta. & VII & $\mathrm{C}$ \\
\hline & Padronizar técnica de venopunção & VII & $\mathrm{C}$ \\
\hline Protocolos & Fazer uso de protocolo para manejo de extravasamento de quimioterapia. & $\mathrm{VI}$ & C \\
\hline
\end{tabular}

QUADRO 3 - Evidências para tratamento de extravasamento de antineoplásicos. Teresina-PI, 2016.

\begin{tabular}{|c|c|c|c|c|}
\hline Temas & Antineoplásicos & Recomendações & Evidência & $\mathrm{GR}^{*}$ \\
\hline \multirow{6}{*}{ Ações imediatas } & \multirow{4}{*}{$\begin{array}{l}\text { Vesicantes, irritantes e não } \\
\text { vesicantes }\end{array}$} & $\begin{array}{l}\text { Suspenção imediata da infusão quando há suspeita ou } \\
\text { confirmação do extravasamento }\end{array}$ & $\mathrm{VI}$ & C \\
\hline & & Estimar a quantidade extravasada & $\mathrm{VI}$ & $\mathrm{C}$ \\
\hline & & Mensurar a área do extravasamento & $\mathrm{VI}$ & C \\
\hline & & Registrar área do extravasamento com foto & $\mathrm{VI}$ & $\mathrm{C}$ \\
\hline & Não vesicantes & $\begin{array}{l}\text { Realizar raio X para análise de localização de cateter } \\
\text { totalmente implantado }(C T I)\end{array}$ & $\mathrm{VI}$ & C \\
\hline & Cisplatina & Remoção cirúrgica de CTI & $\mathrm{VI}$ & $\mathrm{C}$ \\
\hline \multirow{6}{*}{ Tratamento físico } & Antraciclinas & \multirow[t]{3}{*}{ Aplicação de compressas frias } & $\mathrm{VI}$ & B \\
\hline & Mitoxantrona & & VI & $\mathrm{B}$ \\
\hline & Dacabarmazina & & VI & C \\
\hline & Vinblastina & Aplicação de compressas quentes & $\mathrm{VI}$ & $\mathrm{C}$ \\
\hline & Vesicantes e irritantes & Subcutaneous wash-out procedure (SWOP) & $\mathrm{VI}$ & $\mathrm{C}$ \\
\hline & Vinorelbine & Utilização terapia à vácuo após desbridamento cirúrgico & $\mathrm{VI}$ & C \\
\hline \multirow{5}{*}{ Tópicos } & Vinorelbine & Uso de gaze impregnada com acetato por três semanas & $\mathrm{VI}$ & C \\
\hline & Flourouracilo, Epirrubicina, & Uso de gaze impregnada com antibiótico por uma semana & $\mathrm{VI}$ & $\mathrm{C}$ \\
\hline & Ciclofosfamida docetaxel ${ }^{\star *}$ & \multirow[b]{2}{*}{ Subcutaneous wash-out procedure (SWOP) } & $\mathrm{VI}$ & C \\
\hline & $\begin{array}{c}\text { Epirrubicina, paclitaxel e } \\
\text { trabectina** }^{* *}\end{array}$ & & $\mathrm{VI}$ & C \\
\hline & Doxorrubicina & Dimetilsufóxido tópico 99\% & $\mathrm{VI}$ & $\mathrm{C}$ \\
\hline \multirow{2}{*}{ Antídotos } & \multirow{2}{*}{ Antraciclinas } & Dexrazoxano (até 6h após o extravasamento) & $\mathrm{VI}$ & $\mathrm{C}$ \\
\hline & & Uso sistêmico de dexrazoxane & VI & C \\
\hline \multirow{2}{*}{$\begin{array}{l}\text { Abordagem } \\
\text { cirúrgica }\end{array}$} & Antraciclinas & \multirow{2}{*}{$\begin{array}{l}\text { Desbridamento cirúrgico seguido de enxerto em caso de } \\
\text { detecção tardia com evolução para necrose tecidual }\end{array}$} & $\mathrm{VI}$ & $\mathrm{C}$ \\
\hline & Vinorelbine & & VI & C \\
\hline
\end{tabular}

Legenda: * Grau de recomendação. ** Ciclo de quimioterapia. 
QUADRO 4 - Tratamentos para extravasamento de antineoplásicos. Teresina-PI, 2016.

\begin{tabular}{|c|c|c|c|c|}
\hline Antineoplásico & $\begin{array}{l}\text { Antídoto/ } \\
\text { Técnica }\end{array}$ & Dose/Terapêutica & \multicolumn{2}{|c|}{$\begin{array}{l}\text { Evidência - } \\
\qquad \mathrm{GR}^{\star \star *}\end{array}$} \\
\hline \multirow{2}{*}{ Vesicantes e irritantes } & \multirow{2}{*}{$\begin{array}{l}\text { Subcutaneous wash-out } \\
\text { procedure (SWOP)* }\end{array}$} & Dorso da mão: 200 ml de Solução fisiológica (SF) a 0,9\%. & $\mathrm{VI}$ & C \\
\hline & & Tórax: $2000 \mathrm{ml}$ de SF a $0,9 \%$. & $\mathrm{VI}$ & $\mathrm{C}$ \\
\hline Vinorelbine & Terapia à vácuo ** & Pressão de 125 mmHg contínuo durante uma semana. & $\mathrm{VI}$ & C \\
\hline \multirow{2}{*}{ Alcaloides da vinca } & Hialuronidase & $\begin{array}{l}\text { Administrar via subcutânea } 1 \mathrm{ml} \text { da solução em cinco injeções de } \\
0,2 \mathrm{ml} \text { (mudar a agulha a cada injeção). }\end{array}$ & VII & C \\
\hline & Compressa quente & $\begin{array}{l}\text { Calor moderado seco local durante } 30 \text { min após a hialurodinase. } \\
\text { Alternativa: } 15 \text { min a cada } 6 \text { horas por } 2 \text { dias. }\end{array}$ & VII & C \\
\hline \multirow[b]{3}{*}{$\begin{array}{c}\text { Antraciclinas: } \\
\text { Doxorrubicina } \\
\text { Daunorubicina } \\
\text { Epirubicina } \\
\text { Idarubicina }\end{array}$} & $\begin{array}{l}\text { Dimetilsufóxido tópico } \\
\qquad 99 \%\end{array}$ & $\begin{array}{l}4 \text { gotas } / 10 \mathrm{~cm}^{2} \text { de superfície cutânea a cada } 8 \mathrm{~h} \text {. E o dobro da área } \\
\text { afetada durante } 7-14 \text { dias. }\end{array}$ & VII & C \\
\hline & Compressa fria & $\begin{array}{l}\text { Frio local durante } 1 \mathrm{~h} \text { a cada } 8 \mathrm{~h} \text { após a aplicação de DSMO, por } 3 \\
\text { dias. }\end{array}$ & VII & C \\
\hline & $\begin{array}{l}\text { Dexrazoxano } \\
\text { Confirmado: } \\
>5 \mathrm{ml} \\
\text { ou Suspeito: > } 10 \mathrm{ml}\end{array}$ & $\begin{array}{l}\text { Infundir no prazo de seis horas de extravasamento. Durante } \\
\text { 1-2 horas por três dias em uma área diferente do que o local de } \\
\text { extravasamento. A dose recomendada é baseada na superfície } \\
\text { corporal: } \\
\text { - Dia 1: } 1000 \mathrm{mg} / \mathrm{m}^{2} \\
\text { - Dia 2: } 1000 \mathrm{mg} / \mathrm{m}^{2} \\
\text { - Dia 3: } 500 \mathrm{mg} / \mathrm{m}^{2}\end{array}$ & VII & C \\
\hline Mecloretamina & $\begin{array}{l}\text { Tiossulfato de sódio a } \\
\qquad 10 \%\end{array}$ & $\begin{array}{l}\text { Injetar } 2 \mathrm{ml} \text { a cada ml de mecloretamina extravasada, subcutânea } \\
\text { ao redor da zona afetada. Trocar de agulha a cada injeção. }\end{array}$ & VII & C \\
\hline Taxanos & \multirow[b]{2}{*}{ Hialuronidase } & \multirow[b]{2}{*}{$\begin{array}{l}\text { Administrar } 250 \mathrm{U} \text { em } 6 \mathrm{ml} \text { de soro fisiológico administradas em } 6 \\
\text { punções subcutâneas ao redor da zona afetada. }\end{array}$} & \multirow[b]{2}{*}{ VII } & \multirow[b]{2}{*}{$\mathrm{C}$} \\
\hline $\begin{array}{l}\text { Etoposide } \\
\text { Teniposide }\end{array}$ & & & & \\
\hline
\end{tabular}

*A quantidade de SF a 0,9 dependerá do local do extravasamento. ** Após desbridamento cirúrgico. *** Grau de recomendação.

mioterapia em Chiba University Hospital, no Japão, evidenciou uma incidência de $0,08 \%$ de extravasamento em uma amostra de 43.557 pacientes. $O$ valor baixo se deve a aplicação de um protocolo de quimioterapia utilizado no ambulatório, em conjunto a uma equipe multidisciplinar e especializada ${ }^{(14)}$.

Este protocolo estabelece o fluxo de atendimento que se inicia pela aprovação da prescrição por um comitê, posteriormente a mesma era chegada pelo farmacêutico. Além do que, na consulta ambulatorial o enfermeiro explicava sobre o procedimento, o extravasamento e eventos adversos. $O$ preparo dos agentes ocorria em câmaras de segurança por farmacêuticos ${ }^{(14)}$.

Estudos apontam que os extravasamentos ocorrem principalmente em veia periférica na fossa cubital, antebraço, e dorso da mão ${ }^{(14-15)}$. Esses dois últimos locais proporcionam mais danos quando ocorre o extravasamento, pois apresentam menos tecido adiposo e muscular, assim, causam maior comprometimento de nervos e tendões, como apontado por uma especialista norte-americana ${ }^{(16)}$.

\section{Fatores de risco}

São diversos os fatores que podem ocasionar um extravasamento. Estes podem se relacionar a condição do cliente em uso de antineoplásico, aos equipamentos utilizados na administração, bem como as propriedades dos agentes antineoplásicos infundidos.

Desse modo, o paciente encontra-se mais propenso à medida que apresenta disfunção endotelial (por avanço da idade, diabetes mellitus, hipertensão, dislipidemia, aterosclerose, insuficiência renal crônica), em casos de radioterapias prévias, fluxo sanguíneo comprometido, infusão de alta pressão (pediatra ou percepção sensorial alterada), pacientes incapazes de descrever a dor (pediatria, percepção sensorial comprometida), e devido à inexperiência da equipe ${ }^{(17)}$.

Nesse contexto, protocolos, Norte-americanos e Europeus, descrevem outras características da rede venosa que favorecem maior risco, como a diminuição da elasticidade, por fragilidade e em veias de pequeno calibre, obesidade, múltiplas punções anteriores, presença de doença de pele disseminada e movimentação do paciente durante a infusão(16-18).

Quanto aos equipamentos utilizados, o risco é inerente ao cateter periférico principalmente pela possibilidade de desposicionamento relacionado à palpação inadvertida no momento da punção que pode causar perfuração da veia, ao uso de cateter agulhado e fixação inadequada ${ }^{(19-16)}$.

Estudos de casos realizados na Holanda, Índia e Itália, apontaram extravasamento em rede venosa periférica de vinorelbine, antraciclinas, vinorelbine, respectivamente. $O$ tratamento variou de acordo com o agente extravasado. Estes estudos evidenciaram a necessidade de prevenção e detecção precoce, caso ocorra, para implementação imediata de medidas terapêuticas ${ }^{(20-21-22)}$.

Os acessos venosos centrais embora mais seguros, 
também oferecem riscos de extravasamento. Para infusão no cateter totalmente implantado é preciso o uso de uma agulha com tamanho e formato adequado, pois agulhas muito curtas ou que provoquem oscilação podem causar extravasamento por desposicionamento. Assim como, quando são inseridos em locais de difícil estabilização, como no abdome. $O$ cateter venoso central oferece risco por ser possível que sua extensão e ponta se curvem ou rompam, bem como pela formação de trombo na ponta ${ }^{(16)}$.

Nesse sentido, na Alemanha, estudo de caso de uma paciente que sofreu extravasamento de antraciclinas, diagnosticado tardiamente, evoluiu para necrose com retirada imediata do cateter e, posteriormente, cirurgia reparadora com enxerto cutâneo ${ }^{23}$. Situação similar ocorreu nos Estados Unidos, uma senhora de 8I anos sofreu extravasamento de trabectina no cateter venoso central, necessitando de desbridamento cirúrgico(24).

Outro fator relevante é que as drogas administradas ocasionam riscos conforme suas propriedades físico-químicas ou solução infundida. Neste sentido, considera-se a capacidade de se ligar diretamente ao ácido desoxirribonucleico (DNA), capacidade de matar células replicantes, de provocar dilatação vascular, existência de $\mathrm{pH}$ fora do intervalo de 5,5-8,5, osmolaridade do plasma maior que $290 \mathrm{mosmol} / \mathrm{l}$, agentes vasoconstritores, infusão de mais de um agente vesicante, vesicantes de ligação de DNA e antineoplásicos compostos por álcool e polietileno-glicol(25-16).

\section{Prevenção do extravasamento}

A prevenção mais eficiente é a aplicação de protocolo institucional que possibilita unificar ações, por meio de uma linguagem padronizada e sistemática, baseada em evidência ${ }^{(18)}$.

Como não há um consenso quanto à escolha mais adequada do cateter, especialistas em oncologia ${ }^{(19-16-18)}$ indicam uma análise multifatorial quanto: a escolha de membro sem restrições e de uma veia preferencialmente calibrosa, não tortuosas; dispositivo periférico compatível com a veia selecionada; avaliação do tempo de infusão; e idade e peculiaridade de cada cliente; recomendado não palpar a veia no momento da punção; conferir a localização do acesso venoso central.

Neste contexto, estudo realizado na Itália buscou validar um instrumento de avaliação de dificuldade de inserção intravenosa, os itens validados descrevem como prevenção não puncionar em veias não visíveis ou não palpáveis, de fino calibre, esclerosadas, tortuosas, móveis e que sofreram múltiplas punções anteriores ${ }^{(15)}$.

Outras recomendações mencionadas em estudo realizado em ambulatório de quimioterapia no Japão ressalta a importância da assistência de enfermagem, na qual deve demandar cuidados quanto à administração de vesicantes gota-a-gota em bomba de infusão, avaliação a cada 30 minutos e preenchimento da ficha de acompanhamento. Aborda ainda que enfermeiro deve pausar a administração cada vez que o paciente for ao banheiro, e checar o retorno venoso antes de reiniciar a infusão ${ }^{(14)}$.

Especialistas na temática recomendam, como prevenção, a lavagem do acesso com 10 a $20 \mathrm{ml}$ de solução fisiológica a $0,9 \%$ entre infusões de drogas diferentes. Bem como, que a infusão de vesicantes em bolus deve ser administrada concomitante a um fluido compatível. Esclarecem que o enfermeiro deve avaliar o aparecimento de sintomas como edema, dor, rubor e infusão lenta e ressaltam a importância de padronizar técnicas nas instituições ${ }^{(18)}$.

\section{Tratamento do extravasamento}

Existem diversas terapêuticas para minimizar os danos causados por um extravasamento. Estas dependem da droga extravasada, do tempo decorrido entre o evento e a detecção, da localização do evento, da quantidade de antineoplásico extravasado, da via de infusão, dentre outros.

Dessa maneira esta revisão elencou métodos terapêuticos, dentre eles: a aplicação de ações imediatas ao diagnóstico do extravasamento, tratamento físico por meio de aplicação de calor e frio, tópicos, antídotos, abordagem cirúrgica e técnica de Subcutaneous wash-out procedure SWOP (procedimento de lavagem subcutânea).

Estudos de $\operatorname{casos}^{(21-26)}$, trabalho retrospectivo(14), especialista $^{(16)}$ e revisão de literatura ${ }^{(27)}$, concordam que ações imediatas podem retardar complicações frente à ocorrência do extravasamento. Sendo necessário cessar a infusão imediatamente a suspeita ou confirmação, identificar o antineoplásico, estimar a quantidade extravasada, mensurar e registrar a área com foto. Essas ações possibilitam tanto a tomada de decisão em relação ao tratamento a ser implementado, quanto promove um acompanhamento adequado.

A aplicação de calor e frio depende da droga extravasada, revisão de literatura publicada em $2012^{(28)}$ e a especialista Schulmeister ${ }^{(29)}$ concordam com aplicações de compressas quentes para alcaloides da vinca e oxaliplatino, e compressas frias para antraciclinas, mitomicina, mitoxantrona, fluorouracilo e cisplatino. Schulmeister ${ }^{(29)}$ acrescenta o uso de compressa fria para mecloretamina e taxanos.

O mecanismo de ação do calor é induzir vasodilatação e, consequentemente, facilitar o aumento da absorção e distribuição sistêmica do agente, causando menos danos locais. A ação das compressas frias baseia-se na indução de vasoconstriç̧ão com consequente diminuição da velocidade de difusão da droga no interior dos tecidos, de modo a reduzir a potencial área de dano tecidual ${ }^{(30-31-16)}$.

Estudo retrospectivo realizado em um Centro de Alta Complexidade em Oncologia no estado de São Paulo, Brasil, evidenciou que dos 216 extravasamentos que 
ocorreram em um período de cinco anos, em 35.475 pacientes submetidos à quimioterapia, houve ausências de registros quando a prescrição de compressas quentes ou frias. Enfatizou que dos oito registros encontrados, três pacientes foram submetidos a tratamentos com compressas inadequadas à droga extravasada ${ }^{(30)}$.

Esses fatos refletem na atuação de enfermagem, que é responsável pela administração das drogas e implementação de cuidados. Visto que, a aplicação inadequada de compressas pode potencializar o dano tecidual, a depender da droga extravasada. Infere-se a necessidade de uma equipe especializada e atualizada e de fluxos de atendimento padronizados.

Outra técnica de tratamento evidenciada nessa revisão foi a Subcutaneous wash-out procedure (SWOP) ou procedimento de lavagem subcutânea. Esta foi utilizada para vesicante e irritantes em situações distintas, como descritas nos estudos de casos ${ }^{(26-6-32-10)}$, apresentou resultados positivos no que diz respeito à prevenção de necrose tecidual.

Uma série de casos analisados no Cancer Center in South East na Inglaterra, com 89 pacientes que sofreram extravasamento em veias periféricas em 10 anos, apontou que a lavagem local com solução salina foi eficiente em todos os casos, apenas um paciente apresentou infecção local que foi devidamente tratada com antibióticos. Nenhum paciente necessitou de desbridamento cirúrgico ou apresentou complicações posteriores( ${ }^{(6)}$.

Contudo, quando se trata de cateter venoso central, como cateter totalmente implantado, complicações após extravasamento são mais recorrentes devido à localização e quantidade maior de droga. Estudo realizado na Áustria com oito pacientes evidenciou que a retirada imediata do cateter seguida da aplicação da SWOP, foram eficientes nos casos com detecção precoce de extravasamento, em três pacientes, nos quais preveniu a necrose tecidual provocando apenas efeitos secundários. Os demais casos, com detecção tardia, necessitaram de desbridamento cirúrgico ${ }^{(32)}$.

Essa técnica foi desenvolvida por cirurgiões plásticos no Reino Unido, para que assim, os enfermeiros pudessem agir prontamente na ocorrência de extravasamento, oferecendo intervenções da forma mais oportuna e menos inconveniente para os pacientes. Consiste na administração subcutânea de solução salina com o intuito de lavar o local e retirar o antineoplásico extravasado. É uma técnica minimamente invasiva, no entanto, requer treinamento da equipe ${ }^{(27)}$.

As demais terapêuticas implementadas são denominadas antídotos, cuja administração depende da droga extravasada. O dimetilsulfoxido a 99\% (DMSO) é indicado como antidoto para extravasamento antraciclinas e mitomicim C, segundo a European Oncology Nursing Society ${ }^{(18)}$, sua aplica- ção é tópica e o mecanismo de ação é baseado no aumento da permeabilidade da pele, promoção de absorção das drogas e eliminação de radicais livres. Em contrapartida, a especialista Schulmeister ${ }^{16}$ refere que sua aplicação tópica não é recomendada como antídoto, pois, necessita de alta concentração para garantia de eficácia e esta não é liberada para tratamento médico nos Estados Unidos.

Outro antídoto utilizado é tiossulfato de sódio. Embora apresente mecanismo de ação ainda não esclarecido, especialistas acreditam que ele possa neutralizar quimicamente espécies reativas de alquilantes e reduzir a produção de radicais que causam lesão tecidual. É indicado para mecloretamina e deve ser imediatamente após o extravasamento por via subcutânea ${ }^{(16-18)}$. Sua utilização para extravasamento de cisplatina é indicado por especialista ${ }^{(33)}$.

A hialuronidase é enzima que modifica a permeabilidade do tecido conjuntivo por meio da hidrólise do ácido hialurônico. Promove a difusão e absorção da droga. É eficaz para prevenção de necrose tecidual por alcaloides da vin$\mathrm{Ca}^{(33-34-31)}$. Ressalta-se que essas drogas compõem um grupo de citostáticos com maior poder vesicante. Nesse sentido, se faz uso da hialuronidase associada à aplicação de calor seco. Em teoria, potencializam o poder de absorção da droga e não apresentam toxicidade ao tratamento(28).

Como já mencionado, existe um grupo de citostáticos que apresentam maior poder vesicantes. Neste grupo estão inclusas as antraciclinas. $O$ extravasamento por essas drogas causa necrose tecidual grave, dessa forma, apresentam grande risco para os pacientes ${ }^{(35)}$. A European Oncology Nursing Society indica, como único antidoto eficaz contra antraciclinas, o dexrazoxano, chamado Savene na Europa e Totec nos Estados Unidos ${ }^{(36)}$.

A eficácia de dexrazoxano foi testada em estudo realizado em Portsmouth, na Inglaterra, dos 12 pacientes que sofreram extravasamento, nenhum necessitou de abordagem cirúrgica, e apenas $25 \%$ deles apresentaram sintomas secundários ${ }^{(37)}$.

\section{CONCLUSÃO}

O extravasamento pode causar lesões teciduais significativas. Portanto a prevenção é a melhor estratégia a ser adotada pela equipe multidisciplinar. Esta revisão infere sobre a importância da avaliação individual do paciente quanto aos fatores de risco, e a implementação de protocolo assistencial que padronize as ações e a linguagem.

Bem como, evidenciou diversos tratamentos a serem utilizados na prática clínica, com devido treinamento da equipe e conforme as indicações de cada instituição. Os estudos encontrados não proporcionam níveis de recomendações elevados, mas reuniram o que há de mais atual quanto à temática. Espera-se que essas evidências possam nortear a práxis de enfermeiros, a fim de melhorias na atenção aos pacientes acometidos por câncer. 


\section{REFERÊNCIAS}

Oliveira BGRB, Nogueira GA, Carvalho MR,Abreu AM. Caracterização dos pacientes com úlcera venosa acompanhados no Ambulatório de Reparo de Feridas. Rev Eletrônica Enferm [Internet]. 20II [acesso em I3 jul 20I4]; I4(I): I5663.Disponível em: http://www.revistas.ufg.br/index.php/fen/ article/view/10322

I.Who. Globocan 2012: Estimated Cancer Incidence, Mortality and Prevalence Worldwide in 2012. International Agency for Research on Cancer.Word Health Organization, 2015.

2. Rueda, SCM; Arenas, ER. Eventos adversos y complicaciones del tratamiento antineoplásico administrados durante la infância. Medicas UIS 20I4;27(3). Disponível em: http:// www.scielo.org.co/scielo.php?script=sci_abstract\&pi$d=S 012 I-03|920| 4000300009$

3. Moura, JWS; Ferreira de Assis, M; Gonçalvez, FAM; Mendes, MLM. Enfermagem e Quimioterapia: Um estudo no Instituto de Medicina Integral Professor Fernando Figueira - IMIP. Ciências biológicas e da saúde 20I2; I (3): I I-20. Disponível em: https://periodicos.set.edu.br/index.php/facipesaude/article/view/I7/2

4. Gozzo, TO; Panobianco MS; Clapis, MJ; Almeida AM. Toxicidade dermatológica em mulheres com câncer de mama submetidas à quimioterapia. Revista Latino-Americana de Enfermagem 2010; 18(4). Disponível em: http://www.scielo. $\mathrm{br} / \mathrm{pdf} / \mathrm{rlae} / \mathrm{v} 18 \mathrm{n} 4 / \mathrm{pt} \_04$

5. Guidelines Implementation Toolkit. Extravasation guidelines. 2007.

6. Harrold, k; Gould D; Drey, N. The efficacy of saline washout technique in the management of exfoliant and vesicant chemotherapy extravasation: a historical case series report. European Journal of Cancer Care 2013;22: 169-178. Disponível em: https://www.ncbi.nlm.nih.gov/pubmed/2332098।

7. Schulmeister, L; Camp-sorrell, D. Chemotherapy extravasation from implanted ports. Oncology Nurses Forum 2000; 27(3).

8. Conselho Federal de Enfermagem. Resolução 210. Dispõe sobre a atuação dos profissionais de Enfermagem que trabalham com quimioterápico antineoplásicos. COFEN, 1988.

9. Conselho Federal de Enfermagem. Resolução 257. Acrescenta dispositivo ao Regulamento aprovado pela Resolução COFEN N ${ }^{\circ} 210 / 98$, facultando ao Enfermeiro o preparo de drogas Quimioterápicas Antineoplásicas, 2001.

10. Pereira, RP; Cardoso, MJSPO; Martins, MACSC. Atitudes e barreiras à prática de enfermagem baseada na evidência em contexto comunitário. Revista de Enfermagem Referência 2012; III(7). Disponível em: http://www.scielo.mec.pt/scielo. php?script=sci_arttext\&pid=S0874-028320I 2000200006

I I. Mendes, KDS; Silveira, RCCP; Galvão, CM. Revisão integrativa: método de pesquisa para a incorporação de evidências na saúde e na enfermagem. Texto \& Contexto Enfermagem 2008; 17(4). Disponível em: http://www.scielo.br/scielo. php?script=sci_arttext\&pid=SO I 04-070720080004000 I 8
12. Melnyk, B M; Fineout-Overholt, E. Evidence-based practice in nursing \& healthcare.A guide to best practice. In Romanzini,AE. Recuperação cirúrgica retardada: análise do conceito. Ribeirão Preto, 2013.

13. National Pressure Ulcer Advisory Panel. European Pressure Ulcer Advisory Panel and Pan Pacific Pressure Injury Alliance. Prevention and Treatment of Pressure Ulcers: Quick Reference Guide. Emily Haesler (ed.). Cambridge Media: Osborne Park, Australia; 2014.

I4. Sakaiada, E; Sekine, I; Iwasawa, S; Kurimoto, R. et. al. Incidence, Risk factors and treatment outcomes of extravasation of cytotoxic agents in an outpatient chemotherapy clinic. Japanese Journal of Clinical Oncology 2014;44(2): I68-I7I. Diponível em: https://www.ncbi.nlm.nih.gov/pubmed/24302758

15. Pagnutti, L; Bin, A; Donato, R; Di Lena, G. et al. Difficult intravenous access tool in patients receiving peripheral chemotherapy: A pilot-validation study. European Journal of Oncology Nursing 2015; p. I-6. Disponível em: https://www. sciencedirect.com/science/article/pii/SI 4623889I5300077

16. Schulmeister, L. Extravasation Management: Clinical Update. Seminars in Oncology Nursing 201 I;27(I):82-90. Disponível em: https://www.ncbi.nlm.nih.gov/pubmed/2 I2557/6

17. Firat, C; Erbatur, S; Aytekin, AH. Management of extravasation injuries: A retrospective study. Journal of Plastic Surgery and Hand Surgery 2013; 47(I):60-65. Disponível em: https://www.ncbi.nlm.nih.gov/pubmed/231 90022

18. Fidalgo, JAP; Fabregat, LG; Cervantes, A; Margulies, A. et al. Management of chemotherapy extravasation: ESMO-EONS Clinical Practice Guidelines. Annals of Oncology 2012;7(23). Disponível em: https://www.esmo.org/Guidelines/Supportive-and-Palliative-Care/Management-of-Chemotherapy-Extravasation

19. Brito, CD; Lima, EDRP. Dispositivo intravascular periférico curto mais seguro para infusão de quimioterápicos antineoplásticos vesicantes: o que a literatura diz. REME - Revista Mineira de Enfermagem 2012; 16(2):275-79. Disponível em: http://portal.revistas.bvs.br/index.php?search=REME\%20 rev.\%20min.\%20enferm\&connector=ET\&lang=pt

20. Heijmen, L;Vehof, HWM; Laarhoven,V. Blistering of the hand in a breast cancer patient. The Netherlands Journal of Medicine $2011 ; 69(2)$.

2I. Revannasiddaiah, S; Pandey, KC; Pant, NK; Shetty, SK. Local toxicity of antracycline extravasation. BMJ Case Report, 20I3. Disponível em: http://casereports.bmj.com/content/20 I 3/bcr-20 I3-0097 I I.full

22. Manganoni, AM; Pavonim, L; Sereni, E; Farisoglio, C. et al. Vinorelbine chemotherapy-induced blistering. The Netherlands Journal of Medicine 2012;70(6). Disponível em: https://www.ncbi.nlm.nih.gov/pubmed/22859426

23. Vasconcelos, I; Schoenegg, W. Massive breast necrosis after extravasation of a full anthracycline cycle. BMJ Case Report, 2013. Disponível em: https://www.ncbi.nlm.nih.gov/ pmc/articles/PMC38222I7/ 
24. Theman, TA; Hartzell, TL; Sinha, I; Polson, K. et al. Recognition of a new chemotherapeutic vesicant: Trabectedin (Ecteinascidin-743) extravasation with skin and soft tissue damage. Journal of Clinical Oncology 2009;27(33). Disponível em: https://www.ncbi.nlm.nih.gov/pubmed/ 9805684

25. Boschi, R; Rostagno, E. Extravasation of Antineoplastic Agents: Prevention and Treatments. Reports Pediatric 2012; 4(3). Disponível em: https://www.ncbi.nlm.nih.gov/pmc/articles/PMC4227315/

26. Steiert, A; Hille, U; Burke,W; Gohritz,A. et al. Subcutaneous wash-out procedure (SWOP) for the treatment of chemotherapeutic extravasations. Journal of Plastic, Reconstructive \& Aesthetic Surgery 201 I;64:240-247. Disponível em: https://www.ncbi.nlm.nih.gov/pubmed/20542747

27. Dougherty, L; Oakley, C.Advanced practice in the management of extravasation. Cancer Nursing Practice 20 I I; I0(5). Disponível em: https://journals.rcni.com/cancer-nursing -practice/advanced-practice-in-the-management-of-extravasation-cnp20I I.06.10.5.16.c8568

28. Conde-Estévez, D; Mateu-de Antonio, J. Actualización del manejo de extravasaciones de agentes citostáticos. Farmacia Hospitalaria 2012;36(I):34-42. Disponível em: http:// www.elsevier.es/es-revista-farmacia-hospitalaria- I 2 I -articulo-actualizacion-del-manejo-extravasaciones-agentes -SII30634311001097

29. Schulmeister, L. Safe Management of chemotherapy: infusion-related complications. linical Journal of oncology nursing 2014;18(3). Disponível em: https://www.ncbi.nlm.nih. gov/pubmed/24867109

30. Adami, N P; Gutiérrez, MGR; Fonseca, SM; Almeida, EPM. Risk management of extravsation of cytostatic drugs at the adult chemotherapy outpatient clinic of a university hospital. Cancer Nursing 2005; 14:876-882. Disponível em: https://www.ncbi.nlm.nih.gov/pubmed//6000102
31. Schulmeister, L. Vesicant chemotherapy - the management of extravasation. Cancer Nursing Practice 2009;8(3). Disponível em: https://journals.rcni.com/cancer-nursing-practice/vesicant-chemotherapy-the-management-of-extravasation-cnp2009.04.8.3.34.c6979

32. Haslik, W; Hacker, S; Felberbauer, FX; Thallinger, C. Port-aCath extravasation of vesicant cytotoxics: surgical options for a care complication of cancer chemotherapy. EJSO 20I5; 4I:378-385. Disponível em: https://www.ncbi.nlm.nih. gov/pubmed/255I5823

33. Schulmeister, L. Vesicant chemotherapy extravasation antidotes and treatments. Clinical Journal of Oncology Nursing 2009; 13(4). Disponível em: https://www.ncbi.nlm.nih.gov/ pubmed/I9648094

34. Wickham, R; Engelking, C; Sauerland, C; Corbi, D. Vesicant Extravasation Part II: Evidence-Based Management and continuing controversies. Oncology Nursing Forum 2006;33(6). Disponível em: https://www.ncbi.nlm.nih.gov/ pubmed/I7/49397

35. Kane, RC; McGuinn Jr, D; Dagher, R; Justice, R. et al. Dexrazoxane (Totect): FDA Review and approval for the treatment of accidental extravasation following intravenous anthracycline chemotherapy. The Oncologist 2008; I3:445450. Disponível em: https://www.ncbi.nlm.nih.gov/pub$\mathrm{med} / \mathrm{I} 8448560$

36. Wengström, Y; Margulies, A. European Oncology Nursing Society extravasation guidelines. European Journal of Oncology Nursing 2008;( 12):357-36I. Disponível em: https:// www.ncbi.nlm.nih.gov/pubmed/I8765210

37. Muthuramalingam, S; Gale, J; Bradbury, J. Dexrazoxane efficacy for anthracycline extravasation: use in UK clinical practice. The Journal of Clinical Practice 2013;67(3):244249. Disponível em: https://www.ncbi.nlm.nih.gov/pub$\operatorname{med} / 2340969$ 